\title{
Neoadjuvant PD-1 blockade in lung cancer: we're not in Kansas anymore
}

\author{
Brian Cohen, M. Blair Marshall \\ Division of Thoracic Surgery, Department of Surgery, Medstar Georgetown University Hospital, Washington, DC, USA \\ Correspondence to: Dr. Brian Cohen, MD. Division of Thoracic Surgery, Department of Surgery, Medstar Georgetown University Hospital, \\ Washington, DC 20007, USA. Email: brian.D.Cohen@gunet.georgetown.edu. \\ Provenance: This is an invited Editorial commissioned by the Section Editor Chen Chen (The Second Xiangya Hospital of Central South University, \\ Changsha, China). \\ Comment on: Forde PM, Chaft JE, Smith KN, et al. Neoadjuvant PD-1 Blockade in Resectable Lung Cancer. N Engl J Med 2018;378:1976-86.
}

Submitted Jul 05, 2018. Accepted for publication Jul 12, 2018.

doi: $10.21037 /$ jtd.2018.07.98

View this article at: http://dx.doi.org/10.21037/jtd.2018.07.98

In the May 24 $4^{\text {th }}, 2018$ issue of The New England Fournal of Medicine, Patrick Forde and colleagues published a pilot study which examined the safety and feasibility of immune therapy to block programmed death 1 (PD-1) proteins by administering neo-adjuvant PD-1 Inhibitor in the setting of resectable non-small cell lung cancer (NSCLC) (1). In addition to demonstrating a lack of serious adverse events or surgical delay, they were also able to reveal a major pathologic response in tumor burden and provide greater insight into the dynamics of any anti-tumor immune response.

The study was carried out at two medical centers among a single group of 22 patients with stage I, II, or IIIA NSCLC which was deemed to be resectable. Forde et al. administered intravenous nivolumab (at $3 \mathrm{mg} / \mathrm{kilogram}$ of body weight) every two weeks prior to surgical resection for two doses. The primary endpoint was safety and feasibility. To this end, patients were monitored for adverse events or delay in surgery. All patients underwent clinical staging, diagnostic evaluation, and then surgical resection of their primary tumor and lymph nodes following treatment. Further data was collected using genomic analysis of pretreatment tumors, post-treatment tumors, and peripheral blood samples to further investigate anti-tumor action of PD-1 inhibitor. Patients were followed for recurrence-free and overall survival. Of the 22 patients who were enrolled in the trial, 20 received two doses of nivolumab and underwent surgical resection as planned, with one patient undergoing resection after one dose, and another deemed ineligible due to a diagnosis of small cell lung carcinoma. There were minimal adverse effects and no logistic delay in planned surgery that would have rendered this treatment unfeasible.

Post-treatment imaging showed a partial response in 2 out of 21 patients, stable disease in 18, and progression in one. However, of the 20 patients who underwent resection, nine patients $(45 \%)$ demonstrated a major pathologic response, with a complete pathologic response in three patients-suggesting that enlargement on preoperative imaging may represent immune-cell activity rather than tumor progression.

Eleven patients provided pretreatment tumor samples for whole-exome gene sequencing and went on to have their tumors resected. Analysis revealed that tumors with a major pathologic response were those with a significantly higher mutational burden. Additionally, the authors were able to demonstrate that nivolumab increased the frequency of T-cell clones, both in the tumor and systemically. They were able to observe the post-treatment maturation of new mutation-associated, neoantigen-specific $\mathrm{T}$-cell clones that were present in the tumor, as well as in lymph nodes both with and without measurable tumor infiltration. This measured immune response may represent the stimulated ability to identify and target new, tumor-specific antigens in vivo after PD-1 inhibitor administration. The involvement of lymphocytes within lymph nodes was an especially encouraging indication that this treatment may provide a durable response, preventing further tumor growth or 
metastasis post-resection.

Forde's work builds on the understanding that genetic mutations within tumors have the potential to be targeted and destroyed by the immune system. However, this pathway is subject to resistance through the down regulation of the $\mathrm{T}$-cell response (2-5). Other studies have demonstrated the safety and transformational effect of immunotherapy in the treatment of a broad range of cancers, including NSCLC, melanoma, and renal cell carcinoma $(6,7)$. Specifically, in the setting of advanced NSCLC, prior studies have demonstrated an overall survival of 14.9 months, with 1-, 2-, and 3-year survival rates of $56 \%, 45 \%$, and $27 \%$, respectively. These are remarkable results in patients who had already shown disease progression after undergoing one to five prior systemic treatment regimens (8).

This current study has particular value in presenting response after neoadjuvant immunotherapy. The overall response rate of $45 \%$ compares favorably to the maximum objective response rate of $22.2 \%$ observed in the setting of adjuvant nivolumab in advanced disease (8), though this comparison should be made with caution given the difference in trial designs and disease burden between groups. The authors site the work of Liu et al. which compare the therapeutic power of neoadjuvant $v s$. adjuvant immunotherapy in mice models, concluding that neoadjuvant administration generates a greater tumorspecific immune response (9). Indeed, this study describes a strong correlation between the pathological response and the mutational burden of the pretreatment tumor. The presence of post-treatment tumor-specific T-cells clones in the resected tumor, lymph nodes, and the peripheral blood, further support the notion that neoadjuvant immunotherapy could lead to a sustained response, a hypothesis that has been supported by additional work in mice populations $(10,11)$. A recurrence free survival of $73 \%$ at 18 months with this treatment modality show promise and warrant additional study. However, this study was not designed with adequate follow up to speak further to longer-term outcomes.

Ford et al. have cleared an import early hurdle for use of this promising treatment modality. To their primary endpoint, they have shown neoadjuvant nivolumab to be safe and feasible in the setting of this small pilot study. The absence of any new or toxic adverse effects is reassuring, while the ability to administer the treatment preoperatively without delay of planned resection is logistically important for the development and investigation into PD-1 Inhibitors' role in any new treatment algorithms.

Beyond feasibility, they were also able to glean promising information into the pathologic response and immunologic mechanism of neoadjuvant nivolumab. Though these results warrant cautious interpretation given the small sample size of this single-arm pilot study, this study provides exciting preliminary evidence of the efficacy of this treatment modality. Additionally, their carefully tested hypotheses, though performed on a limited number of patients and tumor samples, provided insight into the mechanism of a durable response that is consistent with known mechanisms and prior animal models, and may signal the important role that neoadjuvant administration of immunotherapy may play in the future.

There is an obvious need for more research and betterdefined protocols and expectations for PD-1 inhibitors' role in future treatment algorithms for NSCLC. This work plays an important role in establishing neoadjuvant immunotherapy administration as a valuable target for future research, and helps to reassure the safety and feasibility of such work.

\section{Acknowledgements}

None.

\section{Footnote}

Conflicts of Interest: The authors have no conflicts of interest to declare.

\section{References}

1. Forde PM, Chaft JE, Smith KN, et al. Neoadjuvant PD-1 Blockade in Resectable Lung Cancer. N Engl J Med 2018;378:1976-86.

2. Drake CG, Jaffee E, Pardoll DM. Mechanisms of immune evasion by tumors. Adv Immunol 2006;90:51-81.

3. Dong H, Strome SE, Salomao DR, et al. Tumor-associated B7-H1 promotes T-cell apoptosis: a potential mechanism of immune evasion. Nat Med 2002;8:793-800.

4. Rounds A, Kolesar J. Nivolumab for second-line treatment of metastatic squamous non-small-cell lung cancer. Am J Health Syst Pharm 2015;72:1851-5.

5. Keating GM. Nivolumab: a review in advanced squamous non-small cell lung cancer. Drugs 2015;75:1925-34.

6. Brahmer JR, Drake CG, Wollner I, et al. Phase I study of single-agent anti-programmed death-1 (MDX- 
1106) in refractory solid tumors: safety, clinical activity, pharmacodynamics, and immunologic correlates. J Clin Oncol 2010;28:3167-75.

7. Topalian SL, Hodi FS, Brahmer JR, et al. Safety, activity, and immune correlates of anti-PD-1 antibody in cancer. $\mathrm{N}$ Engl J Med 2012;366:2443-54.

8. Gettinger SN, Horn L, Gandhi L, et al. Overall Survival and Long-Term Safety of Nivolumab (Anti-Programmed Death 1 Antibody, BMS-936558, ONO-4538) in Patients With Previously Treated Advanced Non-Small-Cell Lung Cancer. J Clin Oncol 2015;33:2004-12.

Cite this article as: Cohen B, Marshall MB. Neoadjuvant PD-1 blockade in lung cancer: we're not in Kansas anymore. J Thorac Dis 2018;10(8):4686-4688. doi: 10.21037/jtd.2018.07.98
9. Liu J, Blake SJ, Yong MC, et al. Improved Efficacy of Neoadjuvant Compared to Adjuvant Immunotherapy to Eradicate Metastatic Disease. Cancer Discov 2016;6:1382-99.

10. Salmon H, Idoyaga J, Rahman A, et al. Expansion and Activation of CD103(+) Dendritic Cell Progenitors at the Tumor Site Enhances Tumor Responses to Therapeutic PD-L1 and BRAF Inhibition. Immunity 2016;44:924-38.

11. Goldberg MV, Maris CH, Hipkiss EL, et al. Role of PD-1 and its ligand, B7-H1, in early fate decisions of CD8 T cells. Blood 2007;110:186-92. 